\title{
Towards measuring well-being in smart environments
}

\author{
Eija Halkola*, Lauri Lovén, Marta Cortes, Ekaterina Gilman, Susanna Pirttikangas \\ firstname.lastname@oulu.fi \\ FIN-University of Oulu
}

\begin{abstract}
This study discusses measurement of well-being in the context of smart environments. We propose an experimental design which induces variation in an individual's flow, stress, and affect for testing different measurement methods. Both qualitative and quantitative measuring methods are applied, with a variety of wearable sensors (EEG sensor, smart ring, heart rate monitor) and video monitoring. Preliminary results show significant agreement with the test structure in the readings of wearable stress and heart rate sensors. Selfassessments, on the contrary, fail to show significant evidence of the experiment structure, reflecting the difficulty of subjective estimation of short-term stress, flow and affect.
\end{abstract}

\section{CCS CONCEPTS}

- Human-centered computing $\rightarrow$ Ubiquitous computing; Empirical studies in ubiquitous and mobile computing; Empirical studies in $\mathrm{HCI}$.

\section{KEYWORDS}

well-being, sensoring, measurement, smart environment

\section{ACM Reference Format:}

Eija Halkola*, Lauri Lovén, Marta Cortes, Ekaterina Gilman, Susanna Pirttikangas. 2019. Towards measuring well-being in smart environments. In Adjunct Proceedings of the 2019 ACM International Joint Conference on Pervasive and Ubiquitous Computing and the 2019 International Symposium on Wearable Computers (UbiComp/ISWC '19 Adjunct), September 9-13, 2019, London, United Kingdom. ACM, New York, NY, USA, 4 pages. https://doi.org/10.1145/ 3341162.3344839

Permission to make digital or hard copies of all or part of this work for personal or classroom use is granted without fee provided that copies are not made or distributed for profit or commercial advantage and that copies bear this notice and the full citation on the first page. Copyrights for components of this work owned by others than ACM must be honored. Abstracting with credit is permitted. To copy otherwise, or republish, to post on servers or to redistribute to lists, requires prior specific permission and/or a fee. Request permissions from permissions@acm.org.

UbiComp/ISWC '19 Adjunct, September 09-10, 2019, London, Uk

(C) 2019 Association for Computing Machinery.

ACM ISBN 978-1-4503-6869-8/19/09.. \$15.00

https://doi.org/10.1145/3341162.3344839

\section{INTRODUCTION}

Smart environments have become popular for gameplay, leisure, education, and work. When becoming more common and regularly used, developing tools, measurement protocols, and experimental designs to study well-being in such environments becomes a critical research field. Further, it becomes crucial to understand definitions for well-being when designing experiments and tools that can reliably capture user's experiences and feelings.

As smart environments may rely on various sources, understanding the quality and correlations of the different measurement methods becomes important. In this study, to reach on more comprehensive construction of well-being, our aim was thus to design an experiment to 1) induce variation in an individual's flow, stress, and affect, and 2) measure those variations with number of subjective and objective methods. We choose a gameplay as a experimental scenario due to its suitability for the short-time measurements. In this paper, we present a preliminary experimental design to directly capture user's reactions and mental, emotional and physiological states in a smart environment.

Related work. Well-being comprises a long-term evolution of mental, emotional, physical, social, material and professional dimensions, all measured with both qualitative and quantitative methods $[5,16]$. Lovén et al. [9] give the following definition: "Well-being in smart environments is a latent feature of an individual, manifesting as her immediate mental, physical and emotional states". Further, a desirable state of well-being is characterized by: "1) a flow state of mind, 2) low levels of stress and 3) a balance between negative and positive affect" [9]. Given the above definition of well-being, it is possible to test how well various types of quantitative (self-assessment forms) and qualitative measurement methods (wearable sensors, video or audio analysis) are able to capture the changes in an individual's stress, flow and affect. Mental states [1], such as flow, have been detected from neural activity [11, 19].

For observing flow, also heart rate variability, skin conductance level, and cortisol reactivity are proposed as valid methods [21]. Stress has been detected with skin conductivity observations $[7,12]$ and heart rate variability $[4,10]$. Further, positive and negative affect are acknowledged as components of a person's well-being [2]. Video analysis is 
suggested for detecting affect [20], pulse [13] and, consequently, flow and stress.

In context of smart environments, the common approach is to detect activities [15] and number of people in the space [18] by using motion sensors and/or cameras. The usual use cases for such applications are to count visitors in the space, deliver timely services, or provide tracking mechanism i.e. to serve elderly care. Camera-based image data has also been used to detect emotions [6]. With arising number of pervasive sensors [8], like wearables and smartphones, we can consider various target areas for smart environment sensing. However, more extensive validation is required for such systems to suit to well-being related conclusions. This is where we have positioned this paper.

\section{EXPERIMENTAL DESIGN}

The aim of the experiment is to induce changes in the stress level, flow and affect of the test subjects. We build the experiment around gameplay. The test subject is given a video game to play while the game environment is adjusted to induce reactions in the test subject, which are subsequently measured. Video games offer a controlled environment for rich and interactive game play experiences, which can be measured by various means [14]. We selected the Bubbleshooter game $^{1}$ as it is intuitive as well as easy to learn and adjust to induce variation in the test subjects' stress, flow and affect by modifying the speed, difficulty and the strategy settings of the game.

Setting. In a test space, the test subject is placed in front of a gaming set consisting of a large screen and a mouse for control, and instructed to play a video game. Two researchers, situated behind the subject, are monitoring the sensor devices. One researcher is instructing the test subject and adjusting the game settings while the other researcher collects user feedback with queries. One camera is placed in front of the subject, recording all the facial expressions. A second camera is positioned right behind the researchers.

Before the test, researchers explain and give a written material of the experiment procedure to the test subject. The test subjects also have the opportunity to ask questions.

Test procedure. The tests include in total six phases (0-5), refer to Table 1 . In phase 0 , player is able to get familiar with the game by freely playing it. Immersion in the game, as well as flow, is induced with easy game settings. In phase 1, gameplay is interrupted with a Skype call, aimed at reducing flow. During the following phases (2, 3 and 4), we increase the difficulty by adjusting the settings and introduce further interruptions (external control over the mouse) to reduce flow, increase stress and induce negative affect. To further increase stress in phases 3 and 4 , the player is instructed to

\footnotetext{
${ }^{1}$ https://www.bubbleshooter.net/original-bubble-shooter/
}

win, while we adjust the game settings to make it so difficult that the user will certainly lose. Finally, phase 5 allows the subject to cool off with an easy game setting.

Table 1: Experiment phasing

\begin{tabular}{llllll}
\hline Phase & Time $(\mathrm{min})$ & Stress & Flow & Affect & Comments \\
\hline 0 & $3-5$ & low & + & neutral & easy game, warm-up \\
1 & $8-10$ & low & - & neutral & easy game, Skype call while playing \\
2 & $8-10$ & + & - & - & easy game, external control of mouse \\
3 & $5-10$ & + & - & - & difficult game, losing \\
4 & $3-8$ & + & - & - & difficult game, losing \\
5 & $5-10$ & - & + & + & easy game, winning, cool-off \\
\hline
\end{tabular}

Quantitative measurement. An electroencephalograph (EEG) sensor, a smart ring with a skin conductivity (SC), electrodermal activity (EDA) galvanic skin response (GSR) sensors, and a heart rate (HR) monitor were used to directly capture reactions and mental, emotional, and physiological states. Also a video recording system and the EEG measurements were used for measuring the pose of head and body combined with video. The data sets are detailed in Table 2.

A system developed by Valossa company ${ }^{2}$ detected the emotional states (neutral, happiness, fear, pain, surprise, anger, sadness and disgust) on the test subject faces in the recorded videos. Valossa's analysis method is based on a computational, trainable machine learning model, that uses certain reference points on the three-dimensional surface of human face.

Qualitative measurement. Various self-assessment questionnaires are used at multiple stages of the experiment to find the subjective values of stress, flow and affect). The queries include the PANAS [17] (positive, negative affect), and the Self-Assessment Manikin (SAM) (arousal, valence) [3]. Further, we designed a simple self-assessment questionnaire (SSAQ) consisting of three elements (scale indicating overall happiness; stress, flow, negative affect and attention; and flow-related questions).

Synchronization. For time synchronization, a same mobile phone device was used for both MoodMetric ring and heart rate measurements as they share the same time reference. We use tilts of the mobile phone to facilitate the synchronization of the EEG-data and the measurements from the wearables. When an event happens, we tilt the mobile phone to touch an electrode of the EEG cap. This produces two different effects simultaneously: a glitch in the EEG and a marker in the wearables data. Moreover, each marker is numbered consecutively and shown in the mobile screen in order to assist in the synchronization with the video as well. Therefore, the beginning and end of the experiment and the different test phases and interruptions are marked by means of tilts of the mobile phone, in addition to created markers in raw EEG-data with the EmotivPRO software.

${ }^{2}$ https://valossa.com/ 
Table 2: Sensor data sets.

\begin{tabular}{lllll}
\hline Data type & Target & Source & Sample rate & Comments \\
\hline EEG & stress, flow, affect & EPOC Flex & 128 SPS & 32 channels, gel sensors, EmotivPRO control software \\
SC, EDA, GSR & stress & MoodMetric ring & 3 SPS & 1 channel, continuous range in [1.0, 100.0] \\
HR & stress & Polar OH1 heart rate sensor & 1 SPS & \\
Video & stress, affect & Valossa video analysis & 1 SPS & heart rate, emotions by facial expression \\
\hline
\end{tabular}

Finally, video recordings of game activities, gamer's facial expressions and EEG measurements were synchronized within the Open Broadcaster Software (OBS) environment.

\section{PRELIMINARY RESULTS AND DISCUSSION}

Overall, test was conducted with 18 test subjects $(\mathrm{N}=18)$. We analyzed all the self-assesments before, during and after the tests, as well as the last 60 seconds of each test phase on the MoodMetrics stress sensor data. Further, we glanced at the EEG and the heart rate readings of individual test subjects.

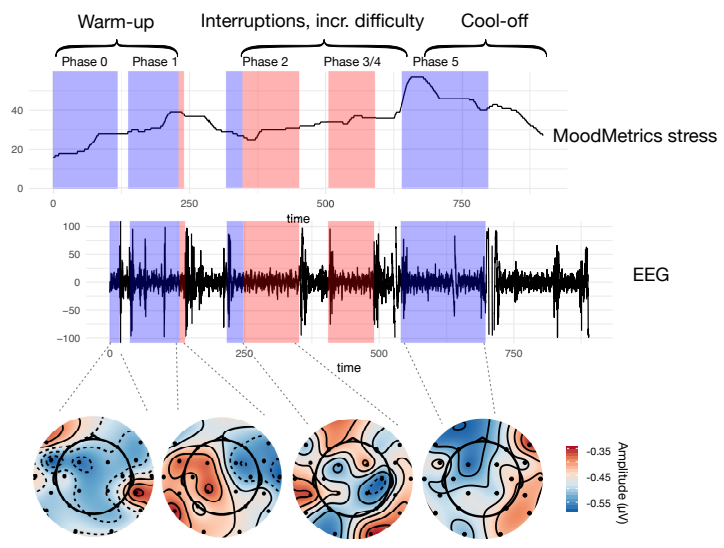

Figure 1: Wearable sensor readings from one test subject. Colored sections in the stress and EEG readings indicate the 6 test phases, with red denoting interruptions or difficulty and blue easiness.

Figure 2, depicting the change in four different affect dimensions from before to after the tests, suggests the test subjects did experience variation in their affect. Figure 1 details the readings from some wearable sensors on one test subject, further suggesting a correspondence between the test structure and the stress readings: as the colored sections in the stress and EEG readings of 1 indicate the 6 test phases, with red denoting interruptions or difficulty and blue easiness, we see a rise in stress over the warm-up period and the difficult phases, and a downward trend during cool-off.

A similar pattern is reflected in the wearable sensor readings in Figure 5 (a). Fitting a linear model with MoodMetrics stress as the response and phase as the categorical covariate results in highly significant estimates of the MoodMetrics
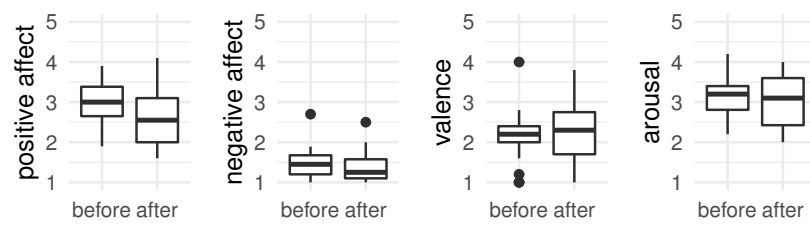

Figure 2: Change in qualitative assessment of affect.

stress values for each phase as seen in Figure 3 (a). Start of the test (phase 0 ) is with high stress, followed by a sharp drop, after which there is a steep upward trend as phases get more difficult and interruptions are introduced. Whereas an identical linear model for heart rate also results in highly significant estimates for each phase (Figure $3(\mathrm{~b})$ ), the pattern follows a generally lowering trend, with phase 3 as the exception with a higher heart rate than previous or next phases.

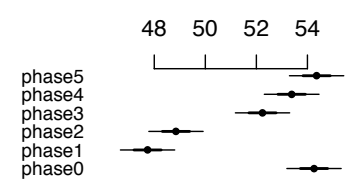

(a)

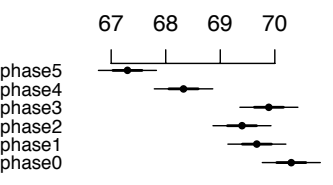

(b)
Figure 3: Estimates of (a) MoodMetrics stress values and (b) Polar heart rate for each phase.

Test self-assessments show a mixed result. Subjective flow appears to exhibit a roughly similar pattern to the MoodMetrics. Subjective stress has a lot of variation, with peaks coinciding with the difficult phases $(3,4)$. Negative affect appears also to peak during the increasingly difficult and annoying parts of the test (phases 2-4). Attention appears to follow a downward trend from initially high values. However, none of these changes turned out significant in statistical testing.

Interestingly, there appeared also no linear correlation between the self-assessment of stress and the MoodMetrics stress value. This could be caused by the relatively low number of test subjects, by a failure in either the MoodMetrics stress values, the self-assessments, or any combination of these. However, as the pair-wise differences of MoodMetrics 

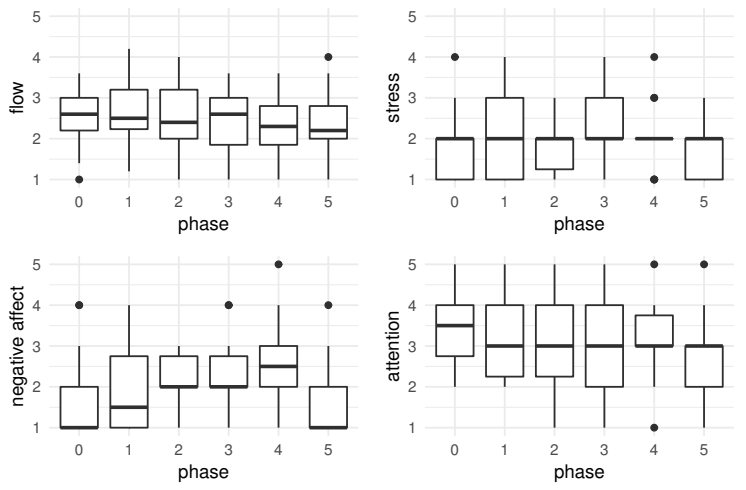

Figure 4: Subjective assessment of flow, stress, and affect.

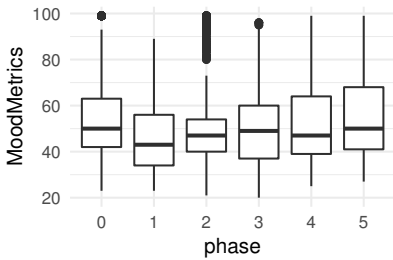

(a)

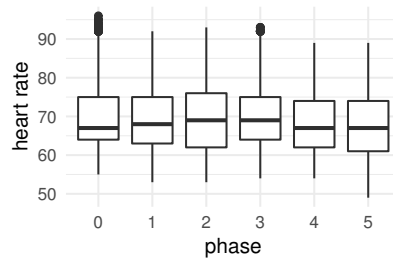

(b)
Figure 5: Averages of MoodMetrics stress and heart rates.

stress values for each phase were significant, while those of the self-assessments were not, the lack of correlation suggests that self-assessment of short-term stress is not feasible.

\section{CONCLUSION}

In this paper, we have identified the research question and outlined a test procedure for examining well-being in smart environments. Further, we have conducted user tests with the proposed gameplay experiment to collect data for further analysis. Preliminary analysis showed the test structure was significantly reflected in the readings of the wearable MoodMetrics and Polar sensors. However, self-assessment questionnaires failed to indicate significant differences between the phases of the experiment, reflecting the difficulty of self-assessment of short-term stress, flow and affect.

In further studies, detailed analysis combining the multimodal measurements will be conducted. The focus of analysis will be on the correlations between the self-assessment concerning the test users' own evaluation of flow, positive and negative affect during game play, as well as the data collected through wearable sensors and the video analysis.

\section{ACKNOWLEDGMENTS}

This work has been funded by Business Finland and the business partners of the VIRPA-D project, Reboot Finland IoT
Factory, Academy of Finland 6Genesis Flagship, the Infotech Oulu, and the Tauno Tönning foundation.

\section{REFERENCES}

[1] Ahern et al. 1985. Differential lateralization for positive and negative emotion in the human brain: EEG spectral analysis. 23, 6 (1985).

[2] Andrews et al. 2012. Social indicators of well-being: Americans' perceptions of life quality. Springer Science \& Business Media.

[3] Bradley et al. 1994. Measuring emotion: the self-assessment manikin and the semantic differential. 7 . of beh. therapy and experimental psychiatry 25, 1 (1994).

[4] Choi et al. 2009. Using heart rate monitors to detect mental stress. In 6th International Workshop on Wearable and Implantable Body Sensor Networks.

[5] Felce et al. 1995. Quality of life: Its definition and measurement. Research in developmental disabilities 16, 1 (1995).

[6] Fernández-Caballero et al. 2016. Smart environment architecture for emotion detection and regulation. f. of biomedical informatics 64 (2016).

[7] Healey et al. 2005. Detecting stress during real-world driving tasks using physiological sensors. IEEE Trans. on intelligent transportation systems 6, 2 (2005).

[8] Kanjo et al. 2015. Emotions in context: examining pervasive affective sensing systems, applications, and analyses. Personal and Ubiquitous Computing 19, 7 (2015).

[9] Lovén et al. 2018. Wellbeing in Smart Environments: Definition, Measurement, Prediction and Control. In Proceedings of the 2018 ACM Int. Foint Conf. and 2018 Int. Symposium on Pervasive and Ubiquitous Computing and Wearable Computers. ACM.

[10] Muaremi et al. 2013. Towards measuring stress with smartphones and wearable devices during workday and sleep. BioNanoScience 3, 2 (2013).

[11] Nakamura et al. 2009. Flow theory and research. Handbook of pos. psyc. (2009).

[12] Picard et al. 1997. Affective wearables. Personal Technologies 1, 4 (1997).

[13] Poh et al. 2010. Non-contact, automated cardiac pulse measurements using video imaging and blind source separation. Optics express 18, 10 (2010).

[14] Palaus et al. 2017. Neural basis of video gaming: A systematic review. Frontiers in human neuroscience 11 (2017).

[15] Rashidi et al. 2010. Discovering activities to recognize and track in a smart environment. IEEE trans. on knowledge and data engineering 23, 4 (2010).

[16] Voorde et al. 2014. Individual well-being and performance at work in the wider context of strategic HRM. In Well-being and performance at work.

[17] Watson et al. 1988. Development and validation of brief measures of positive and negative affect: the PANAS scales. F. of personality and social psychology 54, 6 (1988).

[18] Yu et al. 2006. Human localization via multi-cameras and floor sensors in smart home. In 2006 IEEE Int. Conf. on Systems, Man and Cybernetics, Vol. 5. IEEE.

[19] Yoshida et al. 2014. Brain activity during the flow experience: A functional near-infrared spectroscopy study. Neuroscience letters 573 (2014).

[20] Zeng et al. 2008. A survey of affect recognition methods: Audio, visual, and spontaneous expressions. IEEE trans. on pattern analysis and machine intelligence 31, 1 (2008).

[21] Corinna Peifer. 2012. Psychophysiological correlates of flowexperience. In Advances in flow research. Springer. 